\title{
Modeling of strains and stresses of material nanostructures ${ }^{\S}$
}

\author{
G. SZEFER* and D. JASIŃSKA \\ Institute of Structural Mechanics, Cracow University of Technology, 24 Warszawska St., 31-155 Cracow, Poland
}

\begin{abstract}
Stress and deformation analysis of materials and devices at the nanoscale level are topics of intense research in materials science and mechanics. In these investigations two approaches are observed. First, natural for the atomistic scale description is based on quantum and molecular mechanics. Second, characteristic for the macroscale continuum model description, is modified by constitutive laws taking atomic interactions into account. In the present paper both approaches are presented. For a discrete system of material points (atoms, molecules, clusters), measures of strain and stress, important from the mechanical viewpoint, are given. Numerical examples of crack propagation and deformation of graphite sheets (graphens) illustrate the behavior of the discrete systems.
\end{abstract}

Key words: molecular dynamics, deformation gradients, strain tensor, stress tensor.

\section{Introduction}

The rapid development of nanotechnology associated with the design and manufacture of devices at nano- and microscale level requires thorough analysis of mechanical processes i.e. motion, deformation, stress distribution, yield and failure conditions, in the range of atom and molecule dimensions. Thus the modeling of strains and stresses appearing in nanostructured materials is of great importance and recently constitutes a challenging topic in many fields of science and engineering (mechanics, chemistry, physics, biology, electronics etc.). In the modeling of mechanical properties of nanostructural systems two approaches can be observed. On the one hand, the atomistic description (based on quantum mechanics and molecular dynamics) is extended by notions, typical for the phenomenological mechanics, like deformation gradients, strains, stress tensors, etc [1-5]. On the other hand, the characteristics of the macro scale continuum mechanic descriptions are modified by constitutive laws, which take molecular interactions into account [6-10]. In the present paper a short survey of modeling nanostructured material from the purely mechanical point of view will be given. Both molecular and continuum mechanics description with numerical examples will be presented.

\section{Strains and stresses on the molecular level}

To introduce field quantities in discrete systems, let us consider a system of material points with masses and position vectors $r_{i}, i=1, \ldots, N$ referred to a fixed Cartesian frame $\left\{0, x^{\alpha}\right\}, \alpha=1,2,3$. Let the position of points in the initial configuration be denoted by vectors $\boldsymbol{R}_{i}$. Furthermore, let the collection of all points (atoms, molecules) in the initial and current configurations be denoted by $B_{0}$ and $B_{t}$, respectively. Thus the mapping of $B_{0}$ onto the set $B_{t}$ given by the relation

$$
\boldsymbol{r}_{i j}=\boldsymbol{F}_{i} \cdot \boldsymbol{R}_{i j}
$$

where $\boldsymbol{r}_{i j}=\boldsymbol{r}_{j}-\boldsymbol{r}_{i}, \boldsymbol{R}_{i j}=\boldsymbol{R}_{j}-\boldsymbol{R}_{i}$, defines the molecular deformation gradient tensor $\boldsymbol{F}_{i}$ (assuming that in the neighborhood of the point $A_{i}$ the deformation is homogenous).

This quantity corresponds to the notion known in continuum mechanics, where it constitutes the fundamental measure of deformation coupling the differential line segments $d \boldsymbol{X}$ and $d \boldsymbol{x}$, before and after deformation, respectively. On the atomic (molecular) level different definitions (models) of $\boldsymbol{F}_{i}$ are proposed.

1. The most natural and simple approach, from the mechanical viewpoint, valid for crystal cells, rests on the CauchyBorn hypothesis [11], which states, that crystal cell deforms according to a locally uniform continuum deformation gradient. It means that the lattice vectors behave like material vectors, i.e. they are "embedded" in macroscopic deformation. Thus the Bravais lattice vectors deform according to (1) by the matrix

$$
\boldsymbol{F}=\nabla \boldsymbol{x}=\left(\frac{\partial x^{i}}{\partial X^{K}}\right),
$$

where $x^{i}$ and $X^{K}, i, K=1,2,3$ are the spatial and material coordinates of the continuum particle, respectively. According to the Cauchy-Born rule, the tensor function $\boldsymbol{F}_{i}=\boldsymbol{F}(\boldsymbol{X})$ has a macroscopic meaning and results from the solution of the system of equations of continuum mechanics (obviously obtained by the finite element method).

2. For periodic nanoscale systems, the Parrinello-Rahman model is most frequently used $[2,12,13]$. Thus the calculational cell $\Omega$ (Representative Volume Element RVE) with vectors $\boldsymbol{H}_{1}, \boldsymbol{H}_{2}, \boldsymbol{H}_{3}$, which span the edges of $\Omega$, is introduced (Fig. 1). Forming the matrix $\boldsymbol{H}=\left(H_{\alpha \beta}\right)$, whose columns constitute coordinates of the vectors $\boldsymbol{H}_{\alpha}, \alpha=1,2,3$, one can express the position vector $\boldsymbol{r}_{i}$, related to the corner of $\Omega$ as origin, in terms of this matrix and scaled coordinates $s_{i \alpha}$ as follows $\boldsymbol{r}_{i}=\boldsymbol{H}_{\alpha} s_{i \alpha}=\left(H_{\alpha \beta} s_{i \alpha}\right)=\boldsymbol{H} \cdot \boldsymbol{s}_{i}$ (summation over index $\alpha$ ). Regarding the components $H_{\alpha \beta}$, as general-

\footnotetext{
$\S$ Presented at $1^{\text {st }}$ National Conference of Nano and Micro Mechanics (KKNM08), Krasiczyn, July 2008.

*e-mail: szefer@limba.wil.pk.edu.pl
} 
ized variables and denoting its initial values by $H_{\alpha \beta}^{0}$, one can write

$$
\begin{aligned}
R_{i \beta} & =H_{\alpha \beta}^{0} s_{i \alpha} \Rightarrow s_{i \alpha}=\left(H_{\alpha \beta}^{0}\right)^{-1} R_{i \beta} \\
& \Rightarrow r_{i \beta}=H_{\alpha \beta}\left(H_{\alpha \gamma}^{0}\right)^{-1} R_{i \gamma} .
\end{aligned}
$$

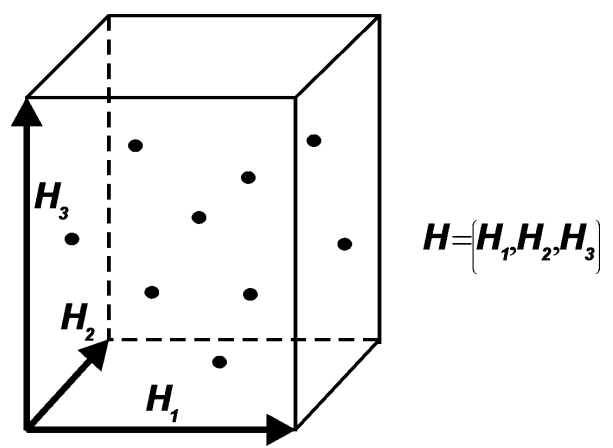

Fig. 1. The Parrinello - Rahman cell

Hence the matrix

$$
\boldsymbol{F}=\boldsymbol{H} \cdot\left(\boldsymbol{H}_{0}\right)^{-1},
$$

which couples the vectors $\boldsymbol{r}_{i j}$ and $\boldsymbol{R}_{i j}$, stands for the deformation gradient of the RVE.

3. In the case of an arbitrary arrangement of the points (without periodicity cell) the atomic strain tensor proposed in [14] is introduced, by using the Voronoi and Delaunay tessellation of space. For any atom $(i)$ the displacement gradient in the form

$$
\nabla \boldsymbol{u}_{i}=\sum_{j=1}^{N_{T}} k_{i j} \frac{\partial \boldsymbol{u}_{i j}}{\partial \boldsymbol{x}}
$$

is defined. Here are: $k_{i j}$ - the volume fraction of Voronoi polyhedron that falls inside the $\mathrm{j}$-th Delaunay tetrahedron, $N_{T}$ - the number of tetrahedrons, $\boldsymbol{u}_{i j}=\boldsymbol{r}_{i j}-\boldsymbol{R}_{i j}$. It is assumed that in the interior of the Voronoi polyhedron the relative displacement $\boldsymbol{u}_{i j}$ is treated as a function of $x^{\alpha}$ in a given Cartesian coordinate system. From (4) it follows that $\boldsymbol{F}_{i}=\mathbf{1}+\nabla \boldsymbol{u}_{i}$ (here $\mathbf{1}$ stands for unity tensor).

4. Another proposal for arbitrary set of points is presented in [15]. This definition takes the form

$$
\boldsymbol{F}_{i}=\left(\sum_{j=1}^{N_{i}} \boldsymbol{r}_{i j} \otimes \boldsymbol{R}_{i j}\right) \cdot\left(\sum_{j=1}^{N_{i}} \boldsymbol{R}_{i j} \otimes \boldsymbol{R}_{i j}\right)^{-1}
$$

as a result of minimization of the function $d\left(\boldsymbol{F}_{i}\right)=$ $\sum_{j=1}^{N_{i}}\left|\boldsymbol{r}_{i j}-\boldsymbol{F}_{i} \boldsymbol{R}_{i j}\right|^{2}$. Here $N_{i}$ means the number of molecules interacting with the atom $(i)$ in the space tesselation. Having $\boldsymbol{F}_{i}$, the strain tensor, defined similarly like in continuum mechanics in the form

$$
\boldsymbol{E}_{i}=\frac{1}{2}\left(\boldsymbol{F}_{i}^{T} \cdot \boldsymbol{F}_{i}-1\right)
$$

can be introduced.

For the control volume $V$ the total tensor $\boldsymbol{E}=\frac{1}{V} \sum_{i} V_{i} \boldsymbol{E}_{i}$ is defined, where the quantity $V_{i}$ stands for the volume of the Voronoi polyhedron of the atom $(i)$.
Especially two scalars: the local dilatation $\varepsilon=$ $\operatorname{tr} \boldsymbol{E}_{i}=E_{\alpha \alpha}^{i}$, and the local deviatoric distortion $\gamma=$ $\left[\frac{2}{3} \operatorname{tr}\left(\boldsymbol{E}_{i}-\frac{1}{3} \varepsilon \mathbf{1}\right)\right]^{\frac{1}{2}}$ are frequently used. After the well known polar decomposition of the deformation gradient $\boldsymbol{F}_{i}=\boldsymbol{Q}_{i} \boldsymbol{U}_{i}$, where $\boldsymbol{U}_{i}$ is the symmetric right hand stretch tensor and $\boldsymbol{Q}_{i}$ is an orthogonal tensor, one obtains $\boldsymbol{U}_{i}^{2}=\boldsymbol{F}_{i}^{T} \boldsymbol{F}_{i}$. With the use of $\boldsymbol{U}_{i}$ the generalized strain measures

$$
\boldsymbol{E}_{m}=\frac{\boldsymbol{U}^{m}-\mathbf{1}}{m}
$$

are defined. For $m=2$ it means the well known strain tensor of Cauchy-Green. The limit for $m \rightarrow 0$ gives the Hencky's logarithmic measure of strain $\boldsymbol{E}=\ln \boldsymbol{U}=\left(\ln \lambda_{k} \delta_{k j}\right)$ (no summation over $\mathrm{k}$ !), where $j, k=1,2,3, \lambda_{k}$ denotes the k-th eigenvalue of $\boldsymbol{U}, \delta_{k j}$ is the Kroneker symbol and brackets stand for a matrix.

In the paper [16] the strain tensor in the form

$$
\hat{\boldsymbol{E}}=\sum_{k=1}^{3} f\left(\lambda_{k}\right) \boldsymbol{w}_{k} \otimes \boldsymbol{w}_{k}
$$

is introduced, where $f\left(\lambda_{k}\right)$ denotes an arbitrary chosen monotonically increasing function, satisfying the conditions $f(1)=0,\left.\frac{d f}{d x}\right|_{x=1}=1$, and $\boldsymbol{w}_{k}$ are the eigenvectors of $\boldsymbol{U}$ Tensor (8) was used in [16] in atomistic simulation of quantum dot.

The most natural way to introduce the notion of stresses at the molecular level is the application of the virial theorem $[17,18]$. Let us consider a system of points with velocities $\boldsymbol{v}_{i}$, subjected to forces resulting from the potential, which depends explicitly on mutual distances $r_{i j}=\left|\boldsymbol{r}_{i j}\right|$. For the system of points in RVE the principle of the dyadic moment of momentum can be written [17]

$$
\dot{\boldsymbol{\kappa}}_{0}=\sum_{i} \frac{1}{V}\left[\sum_{j} \boldsymbol{f}_{i j} \otimes \boldsymbol{r}_{i j}-m_{i} \boldsymbol{v}_{i} \otimes \boldsymbol{v}_{i}\right]
$$

where $\kappa_{0}=\sum_{i} \boldsymbol{r}_{i} \otimes m_{i} \boldsymbol{v}_{i}$ is the tensorial moment of momentum with respect to the origin point $0, \boldsymbol{f}_{i j}=\frac{\partial U}{\partial r_{i j}} \frac{\boldsymbol{r}_{i j}}{r_{i j}}=$ $\chi_{i j} \boldsymbol{r}_{i j}$ is the interaction force, where $\chi_{i j}=\frac{1}{r_{i j}} \frac{\partial U}{\partial r_{i j}}$, and $V$ is the volume of the RVE. Equation (9) expresses the Clausius virial theorem known in statistic mechanics and molecular dynamics (see also [18]).

Hence the expression

$$
\begin{aligned}
& \boldsymbol{\sigma}_{i}=\frac{1}{V}\left[\sum_{j} \boldsymbol{f}_{i j} \otimes \boldsymbol{r}_{i j}-m_{i} \boldsymbol{v}_{i} \otimes \boldsymbol{v}_{i}\right] \\
& =\frac{1}{V}\left[\sum_{j} \chi_{i j} \boldsymbol{r}_{i j} \otimes \boldsymbol{r}_{i j}-m_{i} \boldsymbol{v}_{i} \otimes \boldsymbol{v}_{i}\right],
\end{aligned}
$$

defines the molecular virial stress tensor, which, as one can see, is symmetric. 
The term $\boldsymbol{\sigma}_{i}^{k}=\frac{1}{V} m_{i} \boldsymbol{v}_{i} \otimes \boldsymbol{v}_{i}$ called kinetic tensor, describes the kinetic contribution to the momentum flux (similarly like in continuum mechanics for particles in Eulerian description), whereas the first term defines the tensorial moment of forces.

This tensorial moment, having the meaning of stress, can be obtained also in a different manner, by expressing the potential $U(r)$ by means of strains according to a formula

$$
r_{i j}=\sqrt{\boldsymbol{r}_{i j} \boldsymbol{r}_{i j}}=\sqrt{\left(2 \boldsymbol{E}_{i}+\mathbf{1}\right): \boldsymbol{R}_{i j} \otimes \boldsymbol{R}_{i j}},
$$

hence $U\left(r_{i j}\right)=U\left(\boldsymbol{E}_{i}\right)$. Thus the tensor

$$
\begin{gathered}
\boldsymbol{S}_{i}=\frac{1}{V_{0}} \sum_{j} \frac{\partial U}{\partial \boldsymbol{E}_{j}}=\frac{1}{V_{0}} \sum_{j} \frac{\partial U}{\partial r_{i j}} \frac{\partial r_{i j}}{\partial \boldsymbol{E}_{i}} \\
=\frac{1}{V_{0}} \sum_{j} \frac{1}{r_{i j}} \frac{\partial U}{\partial r_{i j}} \boldsymbol{R}_{i j} \otimes \boldsymbol{R}_{i j}=\frac{1}{V_{0}} \sum_{j} \chi_{i j} \boldsymbol{R}_{i j} \otimes \boldsymbol{R}_{i j}
\end{gathered}
$$

where $V_{0}$ is the volume before deformation, corresponds to the definition of the second Piola-Kirchhoff stress tensor known in elasticity. Similarly the formula

$$
\begin{gathered}
\boldsymbol{T}^{i}=\boldsymbol{S}_{i} \boldsymbol{F}_{i}=\frac{1}{V_{0}} \sum_{j} \frac{1}{r_{i j}} \frac{\partial U}{\partial r_{i j}} \boldsymbol{F}_{i} \cdot \boldsymbol{R}_{i j} \otimes \boldsymbol{R}_{i j} \\
=\frac{1}{V_{0}} \sum_{j} \chi_{i j} \boldsymbol{r}_{i j} \otimes \boldsymbol{R}_{i j}=\frac{1}{V_{0}} \sum_{j} \boldsymbol{f}_{i j} \otimes \boldsymbol{R}_{i j},
\end{gathered}
$$

defines the first Piola-Kirchhoff stress tensor. Multiplying (13) by $J^{-1} \boldsymbol{F}_{i}^{T}$, where $J=\frac{V}{V_{0}}$, one obtains the molecular Cauchy stress tensor

$$
\boldsymbol{\sigma}_{i}^{p}=J^{-1} \boldsymbol{F}_{i}^{T} \boldsymbol{T}_{R}^{i}=\frac{1}{V} \sum_{j} \boldsymbol{f}_{i j} \otimes \boldsymbol{r}_{i j}
$$

which coincides witch the potential part of (10).

In the Parinello-Rahman model the stress tensor appears as a result of the Lagrangian formulation of the equations of motion. It is namely for the system with varying cell

$L=E-U=\sum_{i} \frac{m_{i} \boldsymbol{H} \dot{\boldsymbol{s}}_{i} \cdot \boldsymbol{H} \dot{\boldsymbol{s}}_{i}}{2}+\frac{M \dot{\boldsymbol{H}} \boldsymbol{s}_{i} \cdot \dot{\boldsymbol{H}} \boldsymbol{s}_{i}}{2}-U\left(\boldsymbol{H} \boldsymbol{s}_{i j}\right)$

where $\boldsymbol{H} \dot{\boldsymbol{s}}_{i}$ stands for the molecule velocity $\boldsymbol{v}_{i}, \dot{\boldsymbol{H}} \boldsymbol{s}_{i}$ called steam velocity is the rate of the cell, $\mathrm{M}$ means the mass of RVE, and $\mathrm{E}$ is the kinetic energy of the system. Remark: in this model the cross multiplication terms $\dot{\boldsymbol{H}} \boldsymbol{s}_{i} \cdot \boldsymbol{H} \dot{\boldsymbol{s}}_{i}$ and $\boldsymbol{H} \dot{\boldsymbol{s}}_{i} \cdot \dot{\boldsymbol{H}} \boldsymbol{s}_{i}$ are neglected. Treating the components of the lattice origin vector $\boldsymbol{H}_{\alpha}, H_{\alpha \beta}$, as generalized variables, one can obtain the following expression for the generalized force

$$
\begin{gathered}
\frac{\partial L}{\partial H_{\alpha \beta}}=-\sum_{i}\left(-m_{i} \boldsymbol{H} \dot{\boldsymbol{s}}_{i} \cdot \dot{\boldsymbol{s}}_{i}+\sum_{j} \chi_{i j} \boldsymbol{r}_{i j} \otimes \boldsymbol{s}_{i j}\right) \\
=-\sum_{i}\left[\frac{1}{V}\left(-m_{i} \boldsymbol{H} \dot{\boldsymbol{s}}_{i} \cdot \boldsymbol{H} \dot{\boldsymbol{s}}_{i}+\sum_{j} \chi_{i j} \boldsymbol{r}_{i j} \otimes \boldsymbol{r}_{i j}\right)\right] \boldsymbol{H}^{-1} V .
\end{gathered}
$$

The term inside square brackets corresponds with (10), since $\boldsymbol{s}_{i j}=\boldsymbol{H}^{-1} \boldsymbol{r}_{i j}$, and $\boldsymbol{H}^{-1} V=\boldsymbol{A}$ means the so called "area tensor" (see [19]). Thus we have $\frac{\partial L}{\partial H_{\alpha \beta}}=\sum_{i} \boldsymbol{\sigma}_{i} \cdot \boldsymbol{A}$.

Remark: one should emphasize that the kinetic term $\sigma_{i}^{k}$ in (10) has no effect on true (Cauchy ) stress tensor, since it violates the objectivity rule (when the set of atoms with forces being in equilibrium $\left(\frac{\partial U}{\partial r_{i j}}=0\right)$ moves in rigid motion, all stress components vanished in the ensemble, whereas the kinetic term remains different from zero). More about the virial stresses see also in [20-22].

Extending the Cauchy-Born rule on the higher order deformation gradient in the form (see [23])

$$
\boldsymbol{r}_{i j}=\boldsymbol{F} \boldsymbol{R}_{i j}+\frac{1}{2} \nabla \boldsymbol{F}: \boldsymbol{R}_{i j} \otimes \boldsymbol{R}_{i j}
$$

enables taking into account the local inhomogeneous deformation. The above formula is in correspondence with the quadratic term of Taylor series included in continuum mechanics in the frame of the so called second gradient theory, basing on the expansion $\Delta \boldsymbol{x}=\boldsymbol{F} d \boldsymbol{X}+\frac{1}{2} \nabla \boldsymbol{F}: d \boldsymbol{X} \otimes d \boldsymbol{X}$. In that case the potential takes the form $U\left(\boldsymbol{r}_{i j}\right)=U(\boldsymbol{F}, \nabla \boldsymbol{F})$, and hence

$$
\boldsymbol{T}^{H}=\frac{1}{V_{0}} \frac{\partial U}{\partial \nabla \boldsymbol{F}}=\frac{1}{2 V_{0}} \sum_{j} \boldsymbol{f}_{i j} \otimes \boldsymbol{R}_{i j} \otimes \boldsymbol{R}_{i j}
$$

defines the third order tensor of hyperstresses, whose components describe the intrinsic reaction of the particle system on the inhomogeneous deformation.

In molecular dynamics simulations most frequently the Cauchy microstress tensor (10) is used.

The current position vector $\boldsymbol{r}_{i}$ of the particles results from the solution of the equations of motion, which in the case of two body potentials takes the form

$$
m_{i} \ddot{\boldsymbol{r}}_{i}=\sum_{j} \frac{1}{r_{i j}} \frac{\partial U}{\partial \boldsymbol{r}_{i j}}\left(\boldsymbol{r}_{j}-\boldsymbol{r}_{i}\right)
$$

\section{The continuum atomistic model}

As mentioned in the introduction, the approaches with the mixed continuous - molecular description of nanomaterials are frequently used [24-27]. The idea of this kind of modeling is based on the experimentally verified assumption, that many mechanical properties and behaviors of nanostructures can be effectively described by using continuum mechanics models (e.g. for rods, beams, 2D and 3D continua), provided, that the constitutive laws of the material take into account molecular interactions. The fact, that efficient FEM calculations with molecular material description can be performed, opens new perspectives for investigations in mechanics, material science, and their applications.

Then we have for the material potential $U\left(r_{i j}\right)=$ $W\left(r_{i j}(\boldsymbol{E})\right)=W\left(\sqrt{\left(2 E_{K L}^{i}+\delta_{K L}\right) R_{i j K} R_{i j L}}\right), K L=$ 
$1,2,3\left(R_{i j K}\right.$ are coordinates of the vector $\left.R_{i j}\right)$. Hence

$$
\begin{gathered}
S_{K L}=\frac{1}{V_{0}} \frac{\partial W}{\partial E_{K L}}=\frac{1}{V_{0}} \frac{\partial W}{\partial r_{i j}} \frac{\partial r_{i j}}{\partial E_{K L}} \\
=\frac{1}{V_{0}} \sum_{j} \chi_{i j}\left(r_{i j}\right) R_{i j K} R_{i j L},
\end{gathered}
$$

defines the constitutive equations of the nanomaterial, which coincides with definition (12).

The continuum atomistic equation of motion reads

$$
\begin{gathered}
\left(\frac{1}{V_{0}} \sum_{j} \chi_{i j} R_{i j K} R_{i j L} F_{k L}\right)_{, K}+\rho_{R} b_{K}=\rho_{R} \ddot{\boldsymbol{u}}_{k}, \\
k=1,2,3,
\end{gathered}
$$

where $\rho_{R}$ is the reference density and $($,$) means differentia-$ tion.

Starting with the continuum mechanics solution of the system by expressing the displacements in the obvious FEM form

$$
u_{k}(\boldsymbol{x}, t)=\sum_{\alpha} N_{k \alpha}(\boldsymbol{x}) q_{\alpha}(t)
$$

we obtain the standard matrix equation

$$
M_{\alpha \beta} \ddot{q}_{\beta}=F_{\alpha}^{\mathrm{ext}}-F_{\alpha}^{\mathrm{int}} \text {. }
$$

where

$$
\begin{gathered}
F_{\alpha}^{\mathrm{ext}}=\int_{V} \rho b_{k} N_{k \alpha}(x) d V+\int_{S} p_{k} N_{k \alpha}(x) d S \\
F_{\alpha}^{i n t}=\int_{V} S_{K L}\left(r_{i j}(x)\right) F_{k L} \frac{\partial N_{k \alpha}(x)}{\partial x_{K}} d V \\
M_{\alpha \beta}=\int_{V} \rho N_{k \alpha}(x) N_{k \beta}(x) d V
\end{gathered}
$$

and $\rho$ is density, $b_{k}$ - density of the mass loads, $p_{k}$ - surface tractions (all indexes $K, L$ and $k$ are subject to summation according to the tensor rules).

The term $F_{\alpha}^{\text {int }}$ generally leads to strongly nonlinear structure of the stiffness matrix, due to the material nonlinearity (18). Thus an iterative solution strategy in the form of incremental approach is usually used.

\section{Carbon nanotubes}

As an example of the nanostructure, for modeling of which both molecular and continuous FEM description are often used, we mention CNTs. Since they posses unusual mechanical properties, like the strength $R_{r}=300 \cdot R_{\text {steel }} \cong 150 \mathrm{GPa}$, Young's modulus $E=9 \cdot E_{\text {steel }}$, fraction $a=\frac{R_{r}}{\rho}=12$, where it is $a=2$ for Kevlar, and $a=6-8$ for diamond (for steel as a master fraction $a=1$ ). Properties of CNT depend on chirality, defined by the chirality vector $\boldsymbol{c}=n \boldsymbol{a}_{1}+m \boldsymbol{a}_{2}=\boldsymbol{c}(n, m)$, where $\boldsymbol{a}_{1}, \boldsymbol{a}_{2}$ are basic vectors of the net structure of the CNT. One can distinguish the "zig-zag" structure with $c(n, 0)$, the "armchair" structure with $c(n, n)$, and arbitrary chirality with $\boldsymbol{c}(n, m)$. Lots of experimental data as well as numerical simulation results have been published since CNTs were discovered by Iijima in 1991 [28]. Stiffness coefficient

$$
k(r)=\frac{f(r)}{r-R}=\frac{\frac{\partial U}{\partial r}}{r-R}
$$

and Young's modulus $E_{\mathrm{CNT}}(r)=\frac{1}{r-R} \frac{\partial U}{\partial r} \frac{R}{A}, A=\frac{\pi d^{2}}{4}, d$ - bond diameter, as functions of molecular interactions calculated with the use of the spring and beam models respectively, are given in [29]. More details about modeling of CNTs can be found in [30-35].

\section{Numerical examples}

We end the presentation of modeling of deformations and stresses in nanomaterials by simple examples of numerical simulations. Computations were performed by means of molecular dynamics code LAMMPS [36].

5.1. Crack propagation in the copper sheet. This is a standard numerical test of molecular modeling of stress distribution in nanostructures. We consider a rectangular copper sheet of dimensions $865.36 \times 626.45 \mathrm{~A}^{\circ}$, with an oblique crack obtained by removing 42 atoms from the whole set of 93767 atoms with masses $m_{i}=63.52 \mathrm{~g} / \mathrm{mol}$.The Morse potential $U=D_{0}\left[e^{-2 \alpha\left(r-r_{0}\right)}-2 e^{-\alpha\left(r-r_{0}\right)}\right]$ for $r \leq r_{c}$, with parameters for copper $D_{0}=0.17337 \mathrm{eV}, \alpha=2.320783 \frac{1}{A^{\circ}}$, $r_{0}=2.588754 \mathrm{~A}^{\circ}$ has been used. Positions were defined on a hexagonal lattice. The initial velocities were assigned taking from a Maxwell distribution at a temperature $273 \mathrm{~K}$. The initial equilibrium was reached after 100 steps. Tension in the horizontal direction was induced by the means of velocities $v_{x}=0.0518 \frac{A^{\circ}}{p \mathrm{sec}}$, applied to vertical edges of the sheet. The crack propagation and stress distribution obtained by solving equations (17) and using formula (14) are presented in Figs. 2-5. Stresses presented in Fig. 5 were calculated after "freezing" the system in the final configuration by adding dumping viscous forces proportional to atom's velocity.

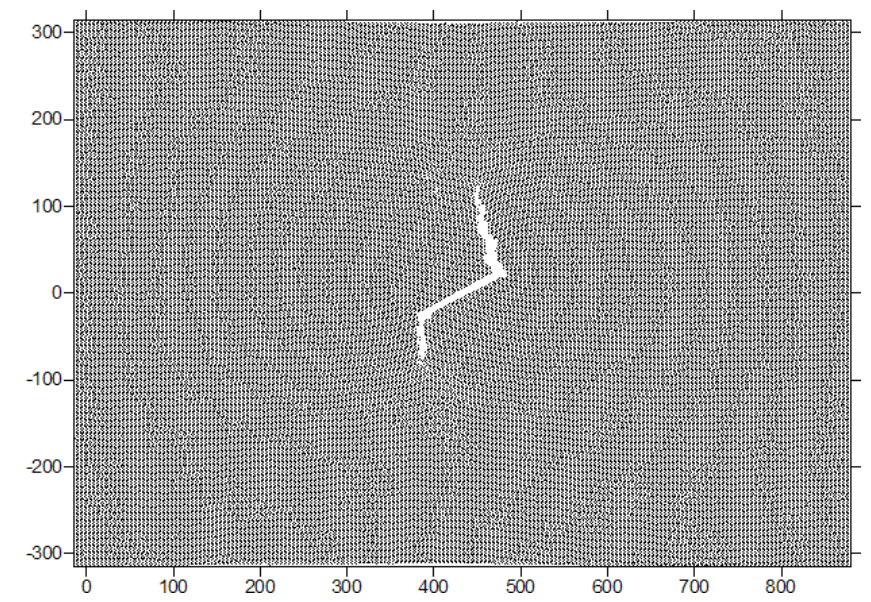

Fig. 2. Deformation at extension 3.3\% 


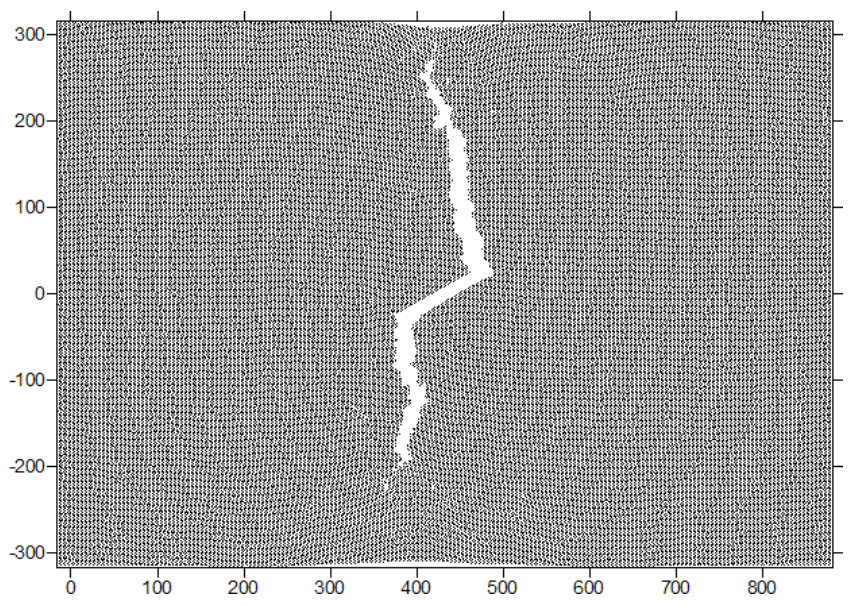

Fig. 3. Deformation at extension $3.7 \%$

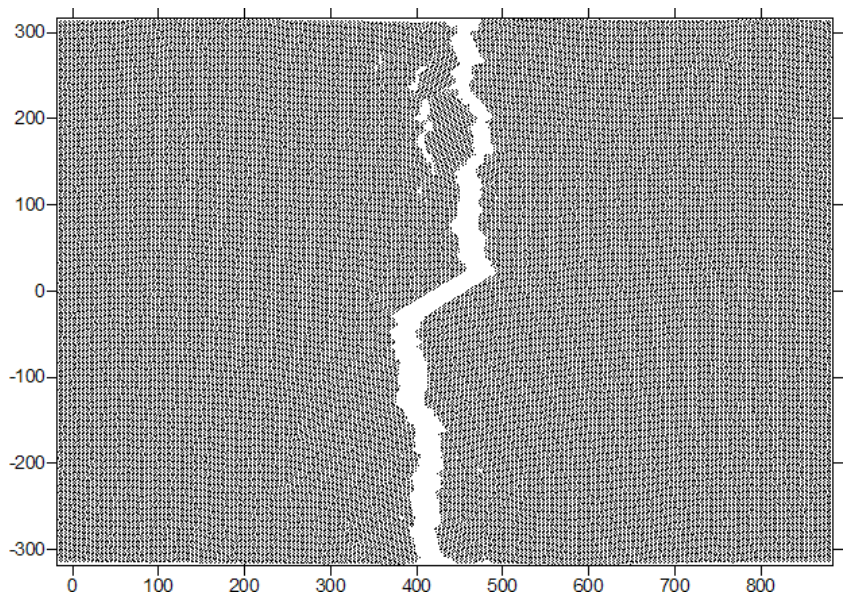

Fig. 4. Deformation at extension $4.1 \%$

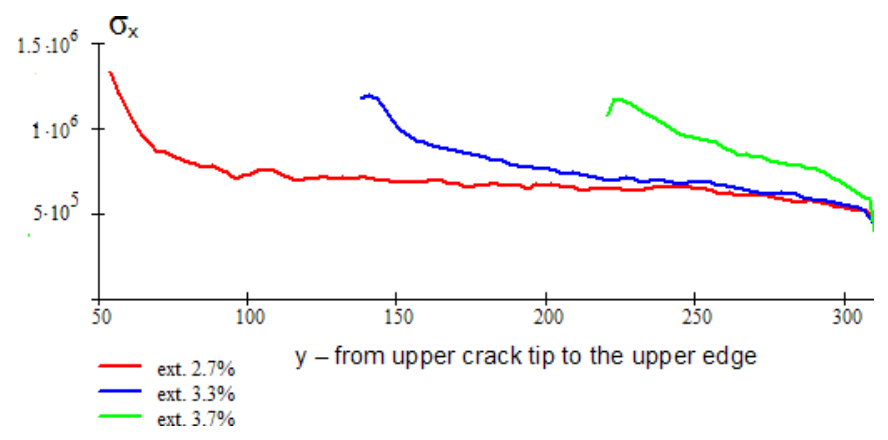

Fig. 5. Stress distribution along the crack propagation direction (from the crack tip to the sheet's edge)

5.2. Graphene sheet and CNT. Graphene sheets of chirality $(6,6)$ and $(10,0)$, and corresponding to them armchair and zig-zag nanotubes have been considered. Morse potential with data $D_{0}=2.894188 \mathrm{eV}, \alpha=2.625 \frac{1}{A^{\circ}}, r_{0}=1.42 \mathrm{~A}^{\circ}$ has been used. Temperature was set to $10 \mathrm{~K}$. Graphene sheets and CNTs have been subjected to tension by applying velocities to vertical edges. Final stretching equals $10 \%$. The resultant deformation are presented in Figs. 6-9.

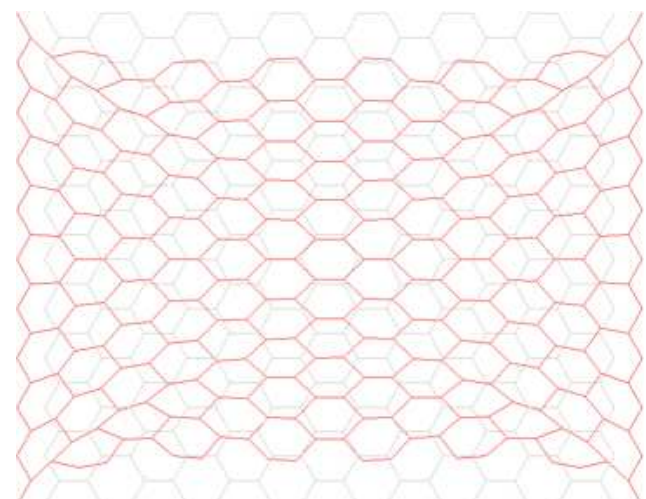

Fig. 6. Deformation of the zigzag graphene

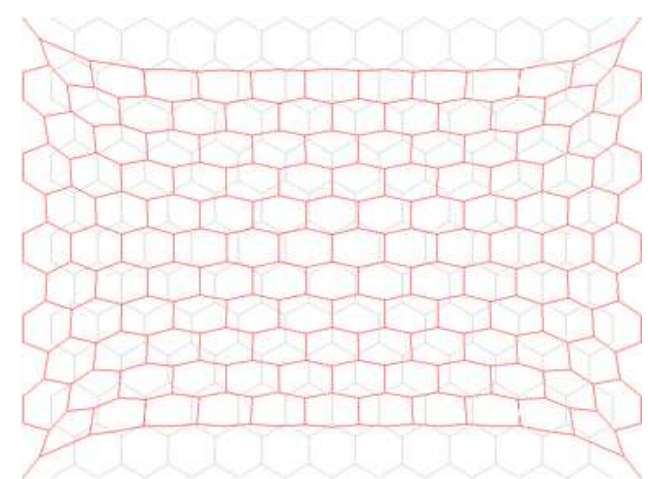

Fig. 7. Deformation of the armchair graphene
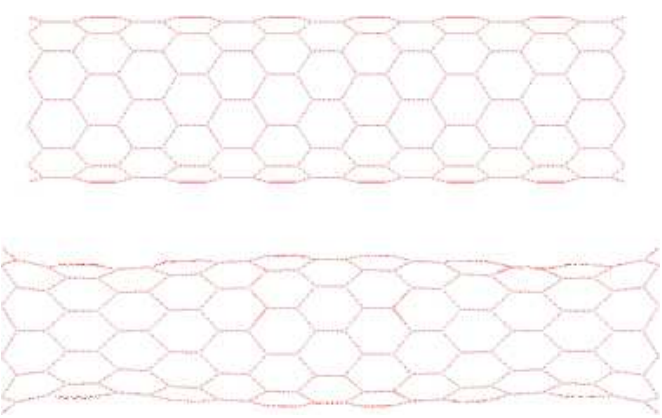

Fig. 8. Zigzag CNT before and after deformation
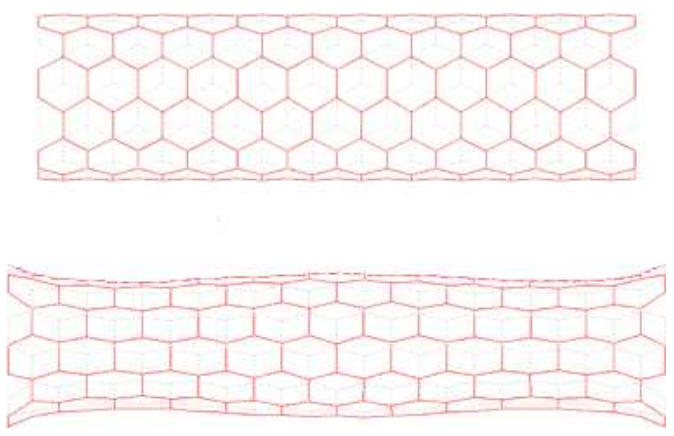

Fig. 9. Armchair CNT before and after deformation

\section{Conclusions}

Two approaches, most frequently used to describe mechanical phenomena at the nanoscale level, were presented. First is the molecular dynamics approach. In frame of this description different deformation measures were shortly demonstrat- 
ed. Analogously to the continuum mechanics three kinds of stress tensors were introduced. The second approach, consistent with continuous description, is modified by formulation of material's constitutive laws taking into account molecular interactions. This proposition, representing pure phenomenological point of view, seems to be very promising for engineering applications, particularly in the case of CNTs and nanocomposites. On this occasion it is worth noting, that according to [25] a CNT composite fibers were manufactured, which were $100 \mathrm{~m}$ long, $50 \mu \mathrm{m}$ in diameter, containing about $60 \%$ SWNTs, and having a tensile strength of 1,8 GPa. Producing of this kind of structural elements with macroscale dimensions is a great challenge for the future engineering. Thus suitable and proper methods of designing and modeling, with nanoscale effects taken into account, are very desirable and up-to-day. Methods presented in this paper can be treated also as a part of multiscale modeling of structures, as well as current extension of investigations in the field of continua with microstructure.

\section{REFERENCES}

[1] J. Ray, "Molecular dynamics equations of motion for systems varying in shape and size", J. Chem. Phys. 79 (10), 5128-5130 (1983).

[2] M. Ribarsky and U. Landman, "Dynamical simulation of stress, strain and finite deformation", Phys. Rev. B 38 (14), 9522-9537 (1988).

[3] W.K. Liu, E. Karpow, and H. Park, Nano Mechanics and Materials, Wiley, Chicester, 2006.

[4] H. Kitagawa, T. Aihara Jr, and Y. Kawazoe, Mesoscopic Dynamics of Fracture, Springer, Berlin, 1998.

[5] D. Raabe, Computational Material Science, Willey, Weinheim, 1998.

[6] Ch. Li and T. Chou, "A structural mechanics approach for the analysis of carbon nanotubes", Int. J. Solids Struct. 40, 24872499 (2003).

[7] X. Gao and K. Li, "Finite deformation continuum model for single-walled carbon nanotubes", Int. J. Solids Struct. 40, 7329-7373 (2003).

[8] A. Pantano, D. Parks, and M. Boyce, "Mechanics of deformation of single- and-multi walled carbon nanotubes", J. Mech. Phys Solids 52, 789-821 (2004).

[9] G. Odagard, T. Gates, L. Nicholson, and K. Wise, "Equivalent continuum modeling of nano-structured materials", Comp. Sci. Techn. 62 (14), 1869-1880 (2002).

[10] K. Tserpes and P. Papanikos, "Finite element modeling of single walled carbon nanotubes", Composites B 36, 468-477 (2005).

[11] R. Phillips, Crystals, Defects, and Microstructures, Cambridge Univ. Press, Cambridge, 2001.

[12] M. Parrinello and A. Rahman, "Crystal structure and pair potentials: a molecular dynamics study", Phys. Rev. Lett. 45 (14), 1196-1199 (1980).

[13] M. Parrinello and A. Rahman, "Strain fluctuations and elastic constants", J. Chem. Phys. 76 (5), 2662-2666 (1982).

[14] P. Mott, A. Argon, and U. Sutter, "The atomic strain tensor", J. Theor. Phys. 101, 140-150 (1992).

[15] R. Pyrz and B. Bochenek, "Discrete-continuum transition at interfaces of nanocomposites", Bull. Pol. Ac.: Tech. 55 (2), 251-260 (2007).
[16] P. Dłużewski and P. Traczykowski, "Numerical simulation of atomic position in quantum dot by means of molecular statics", Arch. Mech. 55 (5-6), 393-406 (2003).

[17] G. Capriz, Introductory remarks to the dynamics of continua with microstructure, Banach Center Publications, vol. 15, PWN, Warsaw, 1986.

[18] W. Hoover, Molecular Dynamics (Lecture Notes in Physics), No. 258, Springer, Berlin, 1986.

[19] U. Landman, W. Luedtke, and E. Ringer, "Molecular dynamics simulations of adhesive contact formation and friction" in Fundamentals of Friction: Macroscopic and Microscopic Processes, eds. I. Singer and H. Polloc, Kluver Ac. Publ., Dordrecht, 1992.

[20] M. Zhou, "A new look at the atomic level virial stress: on continuum-molecular system equivalence", Proc. Roy. Soc. Lond. A 459, 2347-2392 (2003).

[21] H. Wu, "Molecular dynamics study of the mechanics of metal nanovires at finite temperature", Europ. J. Mech. A/Solids 25, 370-377 (2006).

[22] A. Subramaniyan and C. Sun, "Continuum interpretation of virial stress in molecular simulations", Int. J. Solids Struct. 45, 4340-4346 (2008).

[23] R. Sunyk and P. Steinmann, "On higher gradient in continuumatomistic modelling", Int. J. Solids Struct. 40, 6877-6896 (2003).

[24] R. Sunyk and P. Steinman, Zur Beschreibung Komplexen Materialverhaltens, Univ. Stuttgart, 2001.

[25] R. Sumyk and P. Steinman, "Localization analysis of mixed continuum-atomistic model", J. Phys. IV France 11, 251-258 (2001).

[26] T. Belytschko and S. Xiao, "Coupling methods for continuum model with molecular model", Int. J. Multiscale Comput. Engin. 1 (1), 115-126 (2003).

[27] T. Delph, " Local stresses and elastic constants at the atomic scale", Proc. R. Soc. A 461, 1869-1888 (2005).

[28] S. Iijima, "Helical microtubes of graphitic carbon", Nature 354, 56-58 (1991).

[29] M. Chwał, Homogenization of Mechanical Properties of Composite Materials Reinforced by Carbon Nanotubes, $\mathrm{PhD}$ Thesis, Cracow Univ. Technology, Krakow, 2007, (in Polish).

[30] D. Qian, G. Wagner, W. Liu, M. Yu, and R. Ruoff, "Mechanics of carbon nanotubes", Appl. Mech. Rev. 55 (6), 495-533 (2002).

[31] M. Arroyo and T. Belytschko, "A finite deformation membrane based on inter-atomic potentials for the transverse mechanics of nanotubes", Mech. Mater. 35, 193-215 (2003).

[32] M. Arroyo and T. Belytschko, "Finite element methods for the nonlinear mechanics of crystalline sheets and nanotubes", Int. J. Numer. Meth. Eng. 59, 419-456 (2004).

[33] T. Belytschko, S. Xiao, G. Schatz, and R. Ruoff, "Atomistic simulations of nanotube fracture", Phys. Rev. B 65 (23), 235430 (2002).

[34] D. Qian, G. Wagner, and W. Liu, "A multiscale projection method for the analysis of carbon nanotubes", Comp. Meth. Appl. Mech. Engng. 193, 1603-1632 (2004).

[35] K.Chandraseker and S.Mukherjee, "Modification to the Cauchy-Born rule: Applications in the deformation of the single walled carbon nanotubes", Int. J. Solids Struct. 43, 71287144 (2006).

[36] http://lammps.sandia.gov. 